\title{
Outcomes for hospitalized patients with idiopathic pulmonary fibrosis treated with antifibrotic medications
}

Bryan T. Kelly', Viengneesee Thao 2,3 ${ }^{2}$ Timothy M. Dempsey ${ }^{1}$, Lindsey R. Sangaralingham²,3 Stephanie R. Payne ${ }^{2,3}$, Taylor T. Teague1, Teng Moua', Nilay D. Shah ${ }^{2,4}$ and Andrew H. Limper ${ }^{1,2^{*}}$

\begin{abstract}
Background: Idiopathic Pulmonary Fibrosis is a chronic, progressive interstitial lung disease for which there is no cure. However, lung function decline, hospitalizations, and mortality may be reduced with the use of the antifibrotic medications, nintedanib and pirfenidone. Historical outcomes for hospitalized patients with Idiopathic Pulmonary Fibrosis are grim; however there is a paucity of data since the approval of nintedanib and pirfenidone for treatment. In this study, we aimed to determine the effect of nintedanib and pirfenidone on mortality following respiratory-related hospitalizations, intensive care unit (ICU) admission, and mechanical ventilation.
\end{abstract}

Methods: Using a large U.S. insurance database, we created a one-to-one propensity score matched cohort of patients with idiopathic pulmonary fibrosis treated and untreated with an antifibrotic who underwent respiratoryrelated hospitalization between January 1, 2015 and December 31,2018. Mortality was evaluated at 30 days and end of follow-up (up to 2 years). Subgroup analyses were performed for all patients receiving treatment in an ICU and those receiving invasive and non-invasive mechanical ventilation during the index hospitalization.

Results: Antifibrotics were not observed to effect utilization of mechanical ventilation or ICU treatment during the index admission or effect mortality at 30-days. If patients survived hospitalization, mortality was reduced in the treated cohort compared to the untreated cohort when followed up to two years (20.1\% vs $47.8 \%)$.

Conclusions: Treatment with antifibrotic medications does not appear to directly improve 30-day mortality during or after respiratory-related hospitalizations. Post-hospital discharge, however, ongoing antifibrotic treatment was associated with improved long-term survival.

Keywords: Idiopathic pulmonary fibrosis, Antifibrotics, Hospitalization, Critical care, Mechanical ventilation

\section{Background}

Idiopathic Pulmonary Fibrosis (IPF) is a chronic, progressive, fibrosing interstitial pneumonia characterized by progressive dyspnea and deteriorating lung function [1]. IPF occurs predominantly in older adults, particularly in

\footnotetext{
*Correspondence: limper.andrew@mayo.edu

1 Department of Pulmonary and Critical Care Medicine, Mayo Clinic, Gonda 18-South, 200 1st St SW, Rochester, MN 55905, USA

Full list of author information is available at the end of the article
}

men and patients with a history of cigarette smoking, and is defined by histopathologic and/or radiologic pattern of Usual Interstitial Pneumonia (UIP) [1]. The prognosis of IPF is poor overall with reported median survival between 2 and 5 years, though a significant proportion will survive for more than a decade [2,3].

Over the years, various therapies have been studied for the treatment of IPF, yet none were found to offer benefit and recommendations were made against their use 
[4]. In October of 2014, the Federal Drug Administration (FDA) approved the use of nintedanib and pirfenidone for treatment of IPF in the United States (U.S.). At the time of approval, both drugs demonstrated a decrease in the rate of decline of Forced Vital Capacity (FVC) but no mortality benefit, while nintedanib also demonstrated an increase in time to first acute exacerbation (AExIPF) in one of its trial treatment arms $[5,6]$. With these findings, the American Thoracic Society (ATS) clinical practice guidelines were updated, and a conditional recommendation was made for use of the antifibrotic drugs in the treatment of IPF [7]. Given lack of initial data to support a mortality benefit, debate was had as to whether these medications provided enough value for their use given the high costs of treatment and side effects; however, later data obtained from pooled analyses of the antifibrotic drug trials did suggest an additional mortality benefit [8-11]. This data was complemented by that of an Australian IPF Registry [12], and more recently, administrative data from a large United States cohort of commercially insured and Medicare Advantage enrollees which also demonstrated improved mortality on antifibrotic therapy [13].

Despite growing evidence that antifibrotic therapy may improve overall mortality in IPF patients, questions remain as to their impact on specific at-risk subpopulations. One such group of significant interest is patients who are hospitalized, and within that group, those that receive treatment in an Intensive Care Unit (ICU) with and without mechanical ventilation (MV) which may be delivered through both invasive and non-invasive methods. Early data regarding outcomes in these patients demonstrated high mortality [14-18], and the ATS clinical practice guidelines made a weak recommendation against the use of invasive MV in such patients [4]. Since that time, additional data has been published regarding hospitalization and critical illness in IPF patients suggesting lower, albeit still significant mortality [19-23]. A focus on acutely ill and hospitalized patients, however, has not been systematically studied since the availability of antifibrotic therapy. In this study, we utilized a large U.S. administrative claims-based database to evaluate the outcomes of treated and untreated IPF patients hospitalized for acute respiratory-related causes, including those who were cared for in an ICU with or without invasive or non-invasive MV. We hypothesized that antifibrotic therapy prior to hospitalization with an acute respiratory illness may offer a survival advantage compared to untreated patients.

\section{Methods}

Data source

We used deidentified administrative claims data from the OptumLabs Data Warehouse (OLDW). The OLDW contains claims-based information on individuals from all 50 states comprising all ages, ethnicities, and racial groups who are commercially insured or have Medicare Advantage [24]. Since the data are deidentified, this research is exempt from being considered human subjects research by both the Mayo Clinic Institutional Review Board and the NIH. Since the subjects are completely de-identified, it is impossible to re-contact these individuals and hence, the need for additional informed consent is also waived by the Mayo Clinic Institutional Review Board.

\section{Study populations}

We included all adult patients who had their first respiratory hospitalization between January 1, 2015 and December 31, 2018. Respiratory hospitalizations were identified using the following International Classification of Diseases, Ninth Edition (ICD-9) diagnosis codes: 460-466, 470-478, 480-488, 490-496, 500-508, 510-519; and International Classification of Diseases, 10th Edition (ICD-10) diagnosis codes: J00-J06, J09-J18, J20-J22, J30J47, J60-J70, J80-J86, J90-J94, J95.1-J95.8, and J96-J99. A similar approach to studying respiratory hospitalizations in IPF patients with ICD-9 codes has previously been performed [20, 25].

Patients were required to have a diagnosis of IPF (ICD9: 516.31 or ICD-10: J84.112) prior to their index hospitalization and least 6 months of continuous enrollment in their health insurance plan before their hospitalization period. Patients without a diagnosis of IPF were dropped from our analysis. To further increase the accuracy of IPF identification, individuals with rheumatoid arthritis (240.9, 243, 244, 246.1, 246.8, E00-E03, E89.0), sarcoidosis (517.8, I35), and hypersensitivity pneumonitis (495.5, J67.9) were dropped from our analysis, along with individuals younger than 45 years $(\mathrm{N}=48)$. We constructed treated and untreated cohorts of patients with IPF, and defined treated as any patient who filled a prescription for either pirfenidone or nintedanib at least 45 days prior to their index hospitalization. Those who did not fill a prescription for either pirfenidone or nintedanib at least 45 days prior to their index hospitalization were considered not treated. The process of cohort creation is presented in Fig. 1.

\section{Time on treatment}

Patients were considered treated until they stopped filling a prescription for pirfenidone or nintedanib, or if 


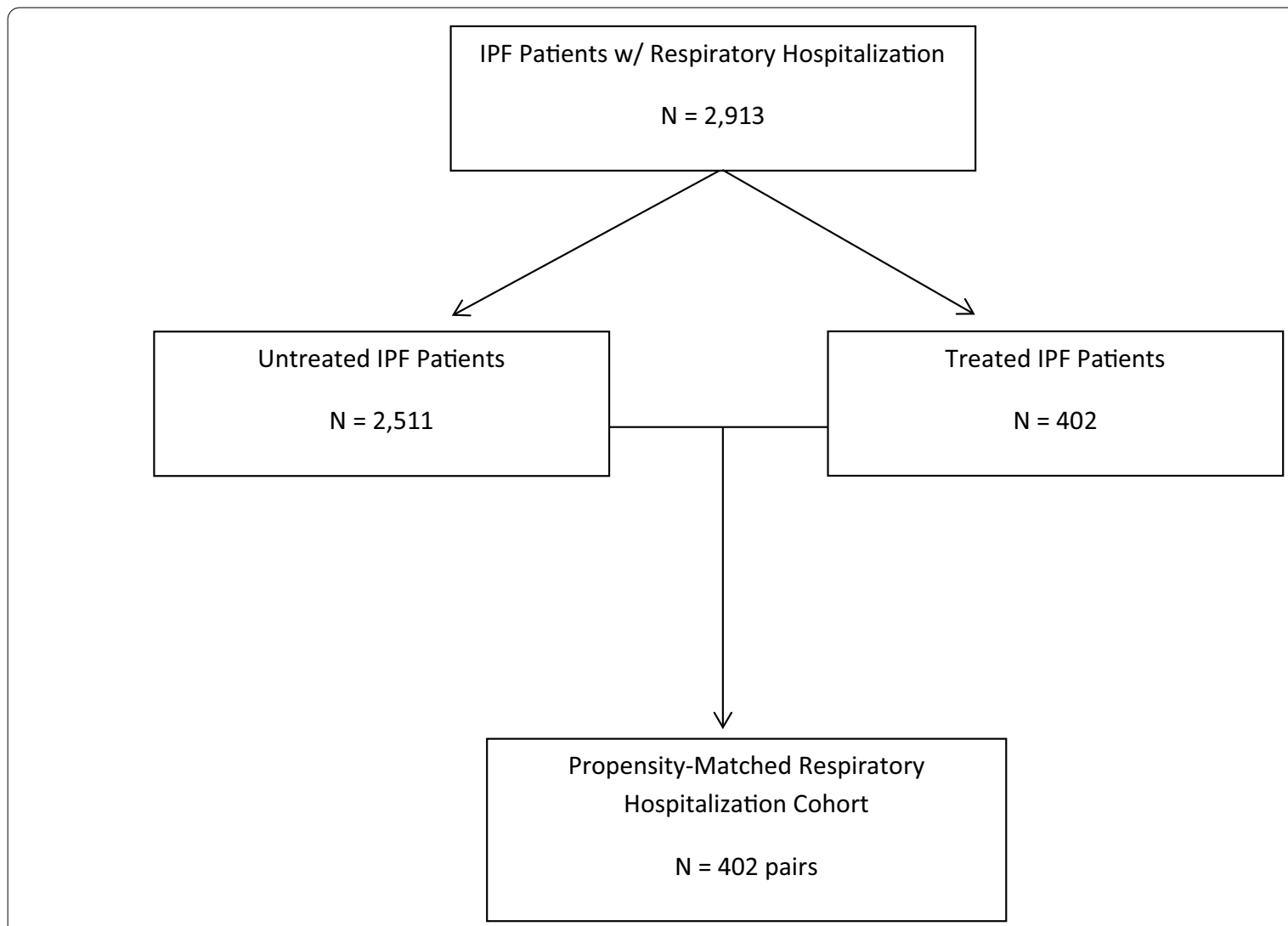

Fig. 1 Generation of propensity-matched cohort of IPF patients with initial respiratory hospitalization

there was a gap of 45 days or greater between their last treatment date and next fill date. We defined last treatment date as 30 days after the last fill date.

\section{Subgroup population}

We repeated the steps outlined previously to create three subgroups, indexed on a first respiratory-related hospitalization to an ICU. These included (1) all ICU respiratory hospitalizations; (2) those who had a respiratory-related ICU hospitalization requiring MV; and (3) those who had a respiratory-related ICU hospitalization without MV (Fig. 2). ICU hospitalizations were identified using revenue codes: 020X-021X. ICU hospitalizations with MV were identified using the revenue codes previously described and Current Procedural Terminology (CPT) codes: 94002-94005; or ICD-9 procedure codes: 96.70-96.72, 93.90; or ICD-10 procedure codes: 5A09357; 5A09457; 5A09557; 5A1935Z; 5A1945Z; 5A1955Z. We also conducted sensitivity analysis by comparing mortality outcomes among non-invasive MV (ICD-9 93.90 ICD-10 5A09357; 5A09457; 5A09557) and invasive MV (ICD-9 96.70-96.72and ICD-10 5A1935Z; 5A1945Z; 5A1955Z).

\section{Independent variables}

We included the following patient demographics: age, sex, race/ethnicity, and census region. In addition, we included the year of index hospitalization and the reason for admission (i.e., primary diagnosis code on the medical claim). Smoking status (ICD-9: 649.0X, 305.1, 989.84, V15.82 and ICD10: F17.X, O00.33X, T65.2X, Z53.01, Z71.6, Z72.0, Z87.891), corticosteroid and oxygen use (CPT: E0424, E0425, E0430, E0431, E0433-E0435, E0440-E0447, E0455, E1352-E1354, E1356-E1359, E1391, E1392), and pulmonologist office visit (CPT: 9920199205; 99211-99215; 99241-99245 with a specialty of 'Pulmonary Disease' listed), prior to the index hospitalization were also captured using their respective billing codes. Hospitalizations prior to the index hospitalization were also accounted for. Comorbidities were assessed using the Elixhauser comorbidity index and were captured with ICD-9 and ICD-10 diagnoses codes prior to the index hospitalization [26]. Prior data has shown that the Elixhauser comorbidity index correlates better with mortality than the Charlson comorbidity index in hospitalized patients with interstitial lung disease [27]. 


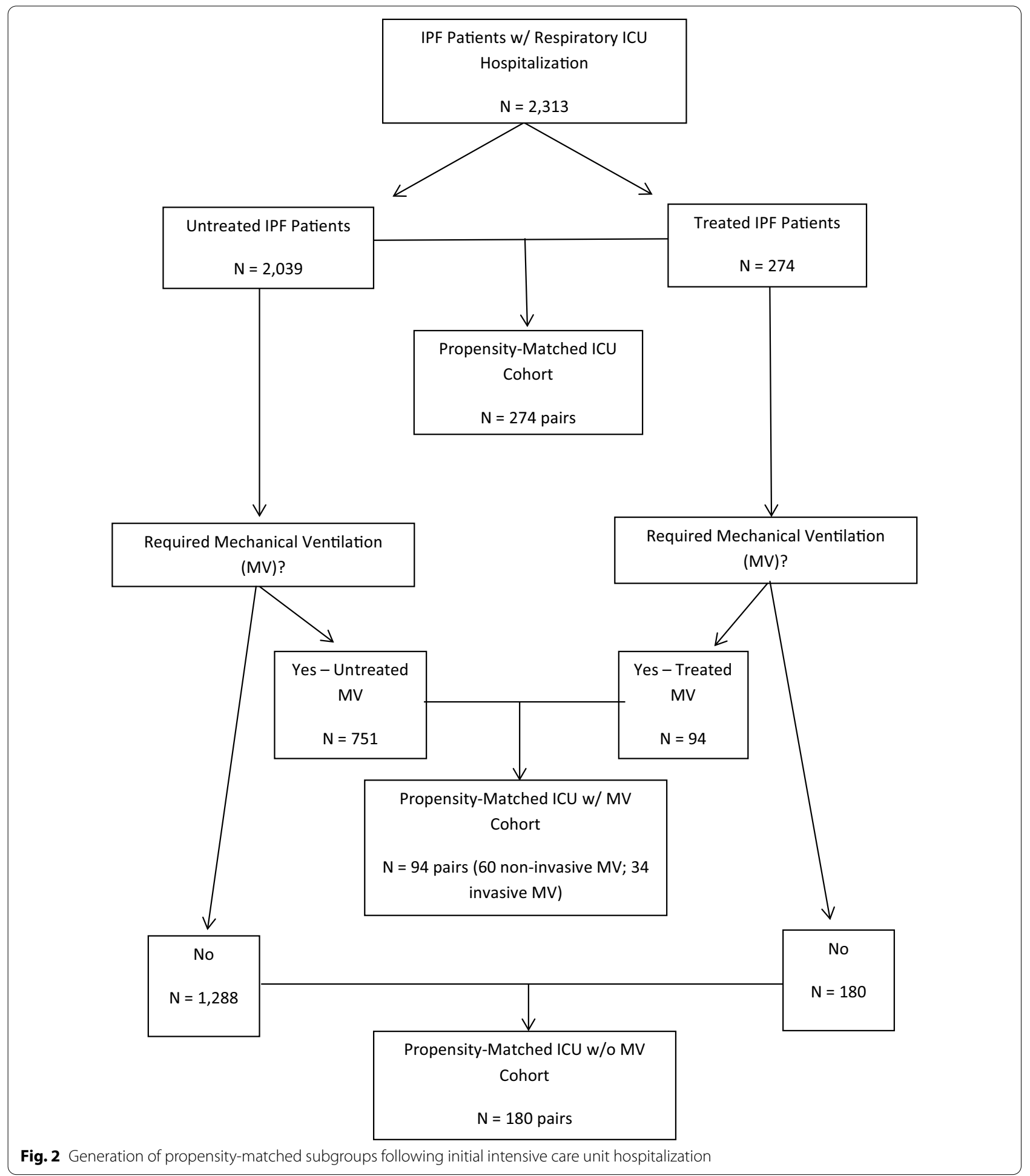

\section{Follow-up}

Follow-up, in the treated cohort, started from the index hospitalization date and continued until the last treatment date, last date of enrollment in the health plan, death date, or end of the study period (April 1, 2019).
Follow-up, in the untreated cohort, started from the index hospitalization date and continued until the last date of enrollment in health plan, death date, end of the study period, or start of pirfenidone or nintedanib. 


\section{Study outcomes}

The primary study outcome was all-cause mortality at 30 days from admission and at the end of follow-up. There are four main sources of mortality information in the OLDW: (1) the Social Security Administration Death Master; (2) electronic health records with deceased status written in the patient charts or patient family reports of death; (3) death as a reason for disenrollment in the health insurance plan; and (4) death indicated in the inpatient discharge status [28]. In a secondary analysis, we compared the use of ICU and MV for all hospitalized patients with IPF treated with or without antifibrotic therapy.

\section{Statistical analysis}

We used propensity score matching to balance the differences in baseline characteristics between the treated and untreated cohorts. A propensity score was estimated using logistic regression based on age, sex, race, geographic region, year of index, reason for admission, smoking status, steroid and oxygen use, healthcare use (prior hospitalizations and pulmonary office visits), and comorbidities. Specifically, we used one-to-one nearestneighbor caliper matching to match patients based on the logit of the propensity score [29]. We evaluated the standardized difference to assess the balance of covariates after matching, and a standardized difference $\leq 10 \%$ was considered acceptable [30]. When balance was not achieved through propensity score matching, we controlled for the unbalanced variable in the analysis.

We used logistic regression to compare mortality at 30 days from admission. Cox proportional hazards regression was used to compare time to death between treated and untreated patients following hospitalization [31]. A similar approach was used for each of the cohorts in the subgroup analysis, with logistic regression to compare ICU and MV use between treated patients and untreated patients during the index hospitalization. All analyses were conducted using SAS 9.4 (SAS Institute Inc.) and Stata version 15.1 (StataCorp).

\section{Results}

\section{Baseline characteristics}

We identified 402 treated and 2,511 untreated patients with IPF, who had a respiratory-related hospitalization between January 1, 2015 and December 31, 2018.The propensity-matched cohort included 402 matched pairs of treated and untreated patients (Table 1, Fig. 1). We then identified 274 treated and 2,039 untreated patients with IPF, who had an ICU hospitalization between January 1, 2015 and December 31, 2018 and created a propensitymatched cohort for all ICU hospitalizations consisting of 274 matched pairs of treated and untreated patients
(Table 2, Fig. 2). Of those requiring an ICU hospitalization with $\mathrm{MV}$, we identified 94 treated and 751 untreated patients with IPF and created a propensity-matched cohort consisting of 94 matched pairs (Table 3, Fig. 2). Of the 94 matched pairs, 34 required invasive $\mathrm{MV}$ and 60 required non-invasive $\mathrm{MV}$. Of those requiring an ICU hospitalization without MV, we identified 180 treated and 1288 untreated patients with IPF and created a propensity-matched cohort of 180 matched (Table 4, Fig. 2).

After propensity matching, baseline characteristics were well balanced between the respiratory-related hospitalization cohorts as shown in Table 1. Furthermore, baseline characteristics were well balanced between the ICU MV subgroups as presented in Tables 2 and 4, respectively. Baseline characteristics within the MV subgroup were well balanced with the exception of: age, race, census region, baseline comorbidities, number of baseline hospitalizations, and smoking status as shown in Table 3.

\section{All-cause mortality}

There was no difference in 30-day mortality between the treated and untreated cohort; $10.0 \%$ vs. $10.2 \%$, hazard ratio (HR) 0.96 , 95\% confidence interval (CI) 0.70-1.33, p value $=0.812$, however antifibrotic treatment prior to any hospitalization was associated with a lower risk of allcause mortality [treated cohort: 39 per 100 person-years vs untreated cohort: 55 per 100 person-years; HR: 0.59, 95\% CI $0.45-0.77, p<0.001]$ when followed through the first 2 years of follow-up as shown in Table 5 and Fig. 3.

\section{All-cause mortality in the subgroup analysis}

We found no difference in all-cause mortality following ICU hospitalizations at 30 days $[16.4 \%$ treated vs $17.9 \%$ untreated, HR 1.05; CI $0.71-1.58, p$ value $=0.782$ ] or at 2 years [51.1\% treated vs $37.2 \%$ untreated, HR 0.79; CI $0.61-1.02, p$ value $=0.075]$ among those treated vs. not (Table 6). There was also no difference in 30-day mortality following MV [30.9\% treated vs $28.7 \%$ untreated, HR 0.91 ; CI $0.52-1.59, p$ value $=0.734$ ] however antifibrotic treatment was associated with a lower risk of all-cause mortality [70.2\% treated vs $53.2 \%$ untreated, HR 0.64; CI $0.43-0.94, p$ value $=0.021]$. When we repeated the analysis by invasive and non-invasive MV, we found no difference in 30-day mortality and 2 year mortality. We found no difference in 30-day mortality following ICU hospitalizations not requiring $\mathrm{MV}[11.7 \%$ treated vs $12.2 \%$ untreated, HR 1.00; CI 0.56-1.83, $p$ value $=0.980]$, or at 2 years $[43.9 \%$ treated vs $28.9 \%$ untreated, HR 0.71 ; CI $0.50-1.00, p$ value $=0.055]$ through 2 years of follow-up. 
Table 1 Baseline demographics of patients with idiopathic pulmonary fibrosis before and after propensity score matching - initial respiratory hospitalizations

\begin{tabular}{|c|c|c|c|c|c|c|}
\hline & \multicolumn{3}{|c|}{ Before propensity score matching } & \multicolumn{3}{|c|}{ After propensity score matching } \\
\hline & No $\operatorname{Rx}(\mathrm{N}=2511)$ & $\begin{array}{l}\text { Pirfenidone/ } \\
\text { Nintedanib } \\
(\mathrm{N}=402)\end{array}$ & Std. Diff & No $R x(N=402)$ & $\begin{array}{l}\text { Pirfenidone/ } \\
\text { Nintedanib } \\
(\mathrm{N}=402)\end{array}$ & Std. Diff \\
\hline \multicolumn{7}{|l|}{ Age group } \\
\hline $45-64$ & 339 (13.5\%) & $53(13.2 \%)$ & -0.009 & 55 (13.7\%) & $53(13.2 \%)$ & -0.015 \\
\hline $65-74$ & 713 (28.4\%) & $163(40.5 \%)$ & 0.258 & $162(40.3 \%)$ & $163(40.5 \%)$ & 0.005 \\
\hline $75+$ & 1459 (58.1\%) & $186(46.3 \%)$ & -0.239 & 185 (46.0\%) & 186 (46.3\%) & 0.005 \\
\hline \multicolumn{7}{|l|}{ Gender } \\
\hline Female & 1182 (47.1\%) & 125 (31.1\%) & -0.332 & $132(32.8 \%)$ & 125 (31.1\%) & -0.037 \\
\hline Male & 1329 (52.9\%) & $277(68.9 \%)$ & 0.332 & $270(67.2 \%)$ & 277 (68.9\%) & 0.037 \\
\hline \multicolumn{7}{|l|}{ Race } \\
\hline White & 1676 (66.7\%) & $293(72.9 \%)$ & 0.134 & 290 (72.1\%) & 293 (72.9\%) & 0.017 \\
\hline Black & 365 (14.5\%) & $33(8.2 \%)$ & -0.200 & 40 (10.0\%) & $33(8.2 \%)$ & -0.061 \\
\hline Hispanic & $311(12.4 \%)$ & 47 (11.7\%) & -0.021 & 48 (11.9\%) & $47(11.7 \%)$ & -0.008 \\
\hline Other & 159 (6.3\%) & $29(7.2 \%)$ & 0.035 & $24(6.0 \%)$ & $29(7.2 \%)$ & 0.050 \\
\hline \multicolumn{7}{|l|}{ Census region } \\
\hline Midwest & 704 (28.0\%) & $110(27.4 \%)$ & -0.015 & $103(26.5 \%)$ & 110 (27.4\%) & 0.039 \\
\hline Northeast & 399 (15.9\%) & $56(13.9 \%)$ & -0.055 & 61 (15.2\%) & $56(13.9 \%)$ & -0.035 \\
\hline South & $1184(47.2 \%)$ & $201(50.0 \%)$ & 0.057 & 208 (51.7\%) & 201 (50.0\%) & -0.035 \\
\hline West & 224 (8.9\%) & 35 (8.7\%) & -0.008 & $30(7.5 \%)$ & 35 (8.7\%) & 0.046 \\
\hline \multicolumn{7}{|l|}{ Baseline comorbidities } \\
\hline Cardiac Arrhythmia & $1037(41.3 \%)$ & $129(32.1 \%)$ & -0.192 & $113(28.1 \%)$ & $129(32.1 \%)$ & 0.087 \\
\hline Congestive Heart Failure & $976(38.9 \%)$ & $108(26.9 \%)$ & -0.257 & $97(24.1 \%)$ & 108 (26.9\%) & 0.063 \\
\hline Other Chronic Pulmonary Conditions & $1833(73.0 \%)$ & $260(64.7 \%)$ & -0.180 & $261(65.0 \%)$ & $260(64.7 \%)$ & -0.005 \\
\hline Depression & $501(20.0 \%)$ & $72(17.9 \%)$ & -0.052 & $68(16.9 \%)$ & $72(17.9 \%)$ & 0.026 \\
\hline Diabetes & $973(38.7 \%)$ & $146(36.3 \%)$ & -0.050 & $136(33.8 \%)$ & $146(36.3 \%)$ & 0.052 \\
\hline Hypertension & $1934(77.0 \%)$ & $279(69.4 \%)$ & -0.173 & $275(68.4 \%)$ & 279 (69.4\%) & 0.021 \\
\hline Pulmonary Circulation Disorder & $644(25.6 \%)$ & $112(27.9 \%)$ & 0.050 & $112(27.9 \%)$ & 112 (27.9\%) & 0.000 \\
\hline Renal Failure & $603(24.0 \%)$ & $72(17.9 \%)$ & -0.150 & $70(17.4 \%)$ & $72(17.9 \%)$ & 0.013 \\
\hline Solid Tumor without Metastasis & $347(13.8 \%)$ & $59(14.7 \%)$ & 0.025 & $65(16.2 \%)$ & $59(14.7 \%)$ & -0.041 \\
\hline Valvular Disease & $642(25.6 \%)$ & $70(17.4 \%)$ & -0.199 & $74(18.4 \%)$ & $70(17.4 \%)$ & -0.026 \\
\hline \multicolumn{7}{|l|}{ Elixhauser comorbidity index } \\
\hline Mean (SD) & $5.7(3.4)$ & $4.7(2.8)$ & -0.344 & $4.5(2.9)$ & $4.7(2.8)$ & 0.062 \\
\hline Median & 5.0 & 4.0 & - & 4.0 & 4.0 & - \\
\hline Q1, Q3 & $3.0,8.0$ & $3.0,6.0$ & - & $2.0,6.0$ & $3.0,6.0$ & - \\
\hline \multicolumn{7}{|l|}{ Nhospitalizations in baseline } \\
\hline 0 & $1452(57.8 \%)$ & $315(78.4 \%)$ & 0.452 & $316(78.6 \%)$ & 315 (78.4\%) & -0.006 \\
\hline 1 & $662(26.4 \%)$ & $70(17.4 \%)$ & -0.218 & $73(18.2 \%)$ & $70(17.4 \%)$ & -0.020 \\
\hline $2+$ & $397(15.8 \%)$ & $17(4.2 \%)$ & -0.393 & $13(3.2 \%)$ & $17(4.2 \%)$ & 0.053 \\
\hline \multicolumn{7}{|l|}{ Year of hospitalization } \\
\hline 2015 & $561(22.3 \%)$ & $36(9.0 \%)$ & -0.375 & $37(9.2 \%)$ & $36(9.0 \%)$ & -0.009 \\
\hline 2016 & $604(24.1 \%)$ & $115(28.6 \%)$ & 0.104 & $127(31.6 \%)$ & 115 (28.6\%) & -0.065 \\
\hline 2017 & $661(26.3 \%)$ & $131(32.6 \%)$ & 0.138 & $118(29.4 \%)$ & $131(32.6 \%)$ & 0.070 \\
\hline 2018 & 685 (27.3\%) & $120(29.9 \%)$ & 0.057 & $120(29.9 \%)$ & 120 (29.9\%) & 0.000 \\
\hline \multicolumn{7}{|l|}{ Reason for admission } \\
\hline Diseases of respiratory system & $1393(55.5 \%)$ & $158(39.3 \%)$ & -0.328 & $155(38.6 \%)$ & 158 (39.3\%) & 0.015 \\
\hline Diseases affecting the interstitium & $928(37.0 \%)$ & $239(59.5 \%)$ & 0.462 & $243(60.4 \%)$ & $239(59.5 \%)$ & -0.020 \\
\hline All other reasons & $190(7.6 \%)$ & $5(1.2 \%)$ & -0.312 & $4(1.0 \%)$ & $5(1.2 \%)$ & 0.024 \\
\hline
\end{tabular}


Table 1 (continued)

\begin{tabular}{|c|c|c|c|c|c|c|}
\hline & \multicolumn{3}{|c|}{ Before propensity score matching } & \multicolumn{3}{|c|}{ After propensity score matching } \\
\hline & No $R x(N=2511)$ & $\begin{array}{l}\text { Pirfenidone/ } \\
\text { Nintedanib } \\
(\mathrm{N}=402)\end{array}$ & Std. Diff & No $\mathrm{Rx}(\mathrm{N}=402)$ & $\begin{array}{l}\text { Pirfenidone/ } \\
\text { Nintedanib } \\
(\mathrm{N}=402)\end{array}$ & Std. Diff \\
\hline Pulmonologist visit & $1323(52.7 \%)$ & $323(80.3 \%)$ & 0.613 & $322(80.1 \%)$ & $323(80.3 \%)$ & -0.006 \\
\hline Smoker & $1274(50.7 \%)$ & $198(49.3 \%)$ & -0.030 & 205 (51.1\%) & $198(49.3 \%)$ & -0.035 \\
\hline Steroid use & $1351(53.8 \%)$ & $229(57.0 \%)$ & 0.064 & $227(56.5 \%)$ & $229(57.0 \%)$ & 0.010 \\
\hline Oxygen use & $1482(59.0 \%)$ & $320(79.6 \%)$ & 0.424 & $310(77.1 \%)$ & $320(79.6 \%)$ & 0.006 \\
\hline
\end{tabular}

\section{ICU and MV use}

We found no difference in ICU admissions during initial hospitalization [OR $0.94,95 \%$ CI $0.71-1.24, p$ value $=0.67$ ] between treated and untreated patients (Table 5); and no difference in MV use [OR 1.17, 95\% CI $0.79-1.70, p$ value $=0.44]$ (Table 6$)$.

\section{Discussion}

To our knowledge, this is the first use of real-world data to evaluate the effects of antifibrotics on hospitalization outcomes in patients with IPF. Our findings are unique as previously published data regarding IPF respiratory hospitalizations have not accounted for the impact of antifibrotic therapy, with much of the available data arising from tertiary referral centers where the acuity of cases and the care administered may limit generalizability.

We observed no impact on 30-day mortality following respiratory-related hospitalizations for patients with IPF treated with antifibrotic medications, including those who required ICU care for any reason, with or without MV, compared to a propensity matched untreated cohorts. Similar rates of ICU utilization across the cohort suggested treatment with antifibrotics prior to hospitalization did not reduce the acuity of hospitalizations. However, if patients survived hospitalization, those with ongoing antifibrotic treatment had improved survival compared to their untreated counterparts up to two years (Fig. 3).

As previously observed by Dempsey et al., treatment with antifibrotic medications is associated with a reduction in hospitalizations [13]. Though lacking a direct impact on 30-day mortality following hospitalizations, it might be suggested that antifibrotic therapy indirectly reduced mortality by reducing the number of hospitalizations. Until an intervention that improves outcomes during hospitalization is identified, prevention of decompensations leading to hospitalization remains an important mechanism to improving overall mortality outcomes.

Previous outcomes data for hospitalized IPF patients have shown in-hospital mortality rates ranging from 10.3 to $22.4 \%$, though such studies were done either entirely prior to the wide availability of antifibrotic therapy [19, 21-23] or overlapping the time period before and after approval without specifically focusing on these therapies (Alqalyoobi). Two of these studies were performed using nationwide databases; the first from Rush et al. reviewed data from 2006 to 2012 and found an in-hospital mortality rate of $11.3 \%$ while Durheim et al. found an in-hospital mortality rate of $10.3 \%$ between October 2011 and October 2014 [21, 22]. While reporting similar mortality numbers to these others and our studies, Alqalyoobi et al. reported mortality differences between academic and non-academic institutions which may be of further consideration and interest [23]. An additional study from Brown et al. was a retrospective review of patients hospitalized at a tertiary care center between 1997 and 2012, and found a mortality of $22.4 \%$ [19]. In review, in-hospital mortality rates between the previously published database studies and our 30-day mortality rates were similar, supporting our study observation that antifibrotics do not appear to reduce in-hospital mortality. While our identified mortality differs from that found by Brown et al., it is important to note that this data was taken from a tertiary care center where acuity of cases, care delivered, and diagnostic techniques may differ compared to that in our population which includes care centers of all levels. Another outcome study from a nationwide Japanese database between 2010 and 2013 demonstrated an in-hospital mortality of $23 \%$, twice that of U.S. national database studies [32]. Ethnic differences in IPF are controversial, and ethnicity alone cannot account for this discrepancy; however such factors, as well as exclusion of less severe cases in some East Asian insurance datasets, may partially explain the higher Japanese mortality $[33,34]$. Furthermore, while the use or efficacy of the antifibrotic medications was not reported in this study, it should be noted that these medications were available for use in Japan during this period, further complicating a direct comparison to this population.

Outcomes regarding those receiving ICU care appear more favorable than previously described. Early literature regarding outcomes of critically ill IPF patients involved smaller cohorts from individual tertiary care 
Table 2 Subgroup analysis: baseline demographics of patients with idiopathic pulmonary fibrosis before and after propensity score matching - all intensive care unit respiratory hospitalizations

\begin{tabular}{|c|c|c|c|c|c|c|}
\hline & \multicolumn{3}{|c|}{ Before propensity score matching } & \multicolumn{3}{|c|}{ After propensity score matching } \\
\hline & No $\operatorname{Rx}(\mathrm{N}=2039)$ & $\begin{array}{l}\text { Pirfenidone/ } \\
\text { Nintedanib }(\mathrm{N}=274)\end{array}$ & Std. Diff & No $\operatorname{Rx}(N=274)$ & $\begin{array}{l}\text { Pirfenidone/ } \\
\text { Nintedanib }(\mathrm{N}=274)\end{array}$ & Std. Diff \\
\hline \multicolumn{7}{|l|}{ Age group } \\
\hline $45-64$ & $287(14.1 \%)$ & $39(14.2 \%)$ & 0.006 & $35(12.8 \%)$ & $39(14.2 \%)$ & 0.043 \\
\hline $65-74$ & 589 (28.9\%) & $111(40.5 \%)$ & 0.245 & $116(42.3 \%)$ & 111 (40.5\%) & -0.037 \\
\hline $75+$ & $1163(57.0 \%)$ & $124(45.3 \%)$ & -0.238 & $123(44.9 \%)$ & $124(45.3 \%)$ & 0.007 \\
\hline \multicolumn{7}{|l|}{ Gender } \\
\hline Female & $905(44.4 \%)$ & $78(28.5 \%)$ & -0.336 & $85(31.0 \%)$ & $78(28.5 \%)$ & -0.056 \\
\hline Male & $1132(55.6 \%)$ & $196(71.5 \%)$ & 0.336 & $189(69.0 \%)$ & $196(71.5 \%)$ & 0.056 \\
\hline \multicolumn{7}{|l|}{ Race } \\
\hline White & $1304(64.0 \%)$ & $179(65.3 \%)$ & 0.027 & $175(63.9 \%)$ & $179(65.3 \%)$ & 0.031 \\
\hline Black & $168(8.2 \%)$ & $11(4.0 \%)$ & -0.177 & $13(4.7 \%)$ & $11(4.0 \%)$ & -0.036 \\
\hline Hispanic & $218(10.7 \%)$ & $31(11.3 \%)$ & 0.020 & $27(9.9 \%)$ & $31(11.3 \%)$ & 0.047 \\
\hline Other & $349(17.1 \%)$ & $53(19.3 \%)$ & 0.060 & $59(21.5 \%)$ & $53(19.3 \%)$ & -0.054 \\
\hline \multicolumn{7}{|l|}{ Census region } \\
\hline Midwest & $564(27.7 \%)$ & $71(25.9 \%)$ & -0.040 & $65(23.7 \%)$ & $71(25.9 \%)$ & 0.051 \\
\hline Northeast & $286(14.0 \%)$ & $31(11.3 \%)$ & -0.082 & $38(13.9 \%)$ & $31(11.3 \%)$ & -0.077 \\
\hline South & $1008(49.4 \%)$ & $143(52.2 \%)$ & 0.056 & $142(51.8 \%)$ & $143(52.2 \%)$ & 0.007 \\
\hline West & $181(8.9 \%)$ & $29(10.6 \%)$ & 0.057 & $29(10.6 \%)$ & $29(10.6 \%)$ & 0.000 \\
\hline \multicolumn{7}{|l|}{ Baseline comorbidities } \\
\hline Cardiac Arrhythmia & $892(43.7 \%)$ & $108(39.4 \%)$ & -0.087 & $112(40.9 \%)$ & $108(39.4 \%)$ & -0.030 \\
\hline Congestive Heart Failure & $845(41.4 \%)$ & $87(31.8 \%)$ & -0.201 & $97(35.4 \%)$ & $87(31.8 \%)$ & -0.077 \\
\hline Other Chronic Pulmonary Conditions & $1568(76.9 \%)$ & $180(65.7 \%)$ & -0.250 & $185(67.5 \%)$ & $180(65.7 \%)$ & -0.039 \\
\hline Depression & $407(20.0 \%)$ & $51(18.6 \%)$ & -0.035 & $42(15.3 \%)$ & $51(18.6 \%)$ & 0.087 \\
\hline Diabetes & $819(40.2 \%)$ & $104(38.0 \%)$ & -0.044 & 109 (39.8\%) & $104(38.0 \%)$ & -0.037 \\
\hline Hypertension & $1600(78.5 \%)$ & $194(70.8 \%)$ & -0.176 & $200(73.0 \%)$ & $194(70.8 \%)$ & -0.049 \\
\hline Pulmonary Circulation Disorder & $553(27.1 \%)$ & $86(31.4 \%)$ & 0.093 & $81(29.6 \%)$ & $86(31.4 \%)$ & 0.040 \\
\hline Renal Failure & $486(23.8 \%)$ & $52(19.0 \%)$ & -0.118 & $47(17.2 \%)$ & $52(19.0 \%)$ & 0.047 \\
\hline Solid Tumor without Metastasis & $302(14.8 \%)$ & $40(14.6 \%)$ & -0.006 & $38(13.9 \%)$ & $40(14.6 \%)$ & 0.021 \\
\hline Valvular Disease & $551(27.0 \%)$ & $58(21.2 \%)$ & -0.135 & $59(21.5 \%)$ & $58(21.2 \%)$ & -0.009 \\
\hline \multicolumn{7}{|l|}{ Elixhauser comorbidity index } \\
\hline Mean (SD) & $5.9(3.3)$ & $5.2(2.9)$ & -0.258 & $5.1(3.2)$ & $5.2(2.9)$ & 0.004 \\
\hline Median & 6.0 & 5.0 & - & 5.0 & 5.0 & - \\
\hline Q1, Q3 & $3.0,8.0$ & $3.0,7.0$ & - & $3.0,7.0$ & $3.0,7.0$ & - \\
\hline \multicolumn{7}{|l|}{ Nhospitalizations in baseline } \\
\hline 0 & $1132(55.5 \%)$ & $181(66.1 \%)$ & 0.217 & $178(65.0 \%)$ & $181(66.1 \%)$ & 0.023 \\
\hline 1 & $535(26.2 \%)$ & $67(24.5 \%)$ & -0.042 & $70(25.5 \%)$ & $67(24.5 \%)$ & -0.025 \\
\hline $2+$ & $372(18.2 \%)$ & $26(9.5 \%)$ & -0.255 & $26(9.5 \%)$ & $26(9.5 \%)$ & 0.000 \\
\hline \multicolumn{7}{|l|}{ Year of hospitalization } \\
\hline 2015 & $448(22.0 \%)$ & $30(10.9 \%)$ & -0.301 & $30(10.9 \%)$ & $30(10.9 \%)$ & 0.000 \\
\hline 2016 & $498(24.4 \%)$ & $69(25.2 \%)$ & 0.019 & $72(26.3 \%)$ & $69(25.2 \%)$ & -0.025 \\
\hline 2017 & $532(26.1 \%)$ & $84(30.7 \%)$ & 0.101 & $83(30.3 \%)$ & $84(30.7 \%)$ & 0.008 \\
\hline 2018 & $561(27.5 \%)$ & $91(33.2 \%)$ & 0.124 & $89(32.5 \%)$ & $91(33.2 \%)$ & 0.016 \\
\hline \multicolumn{7}{|l|}{ Reason for admission } \\
\hline Diseases of respiratory system & $1352(66.3 \%)$ & $136(49.6 \%)$ & -0.342 & $136(49.6 \%)$ & $136(49.6 \%)$ & 0.000 \\
\hline Diseases affecting the interstitium & $523(25.6 \%)$ & $132(48.2 \%)$ & 0.479 & $134(48.9 \%)$ & $132(48.2 \%)$ & -0.015 \\
\hline All other reasons & $164(8.0 \%)$ & $6(2.2 \%)$ & -0.268 & $4(1.5 \%)$ & $6(2.2 \%)$ & 0.055 \\
\hline Pulmonologist visit & $1136(55.7 \%)$ & $225(82.1 \%)$ & 0.595 & $230(83.9 \%)$ & $225(82.1 \%)$ & -0.049 \\
\hline Smoker & $1066(52.3 \%)$ & 135 (49.3\%) & -0.060 & $134(48.9 \%)$ & 135 (49.3\%) & 0.007 \\
\hline Steroid use & $1145(56.2 \%)$ & $183(66.8 \%)$ & 0.219 & $188(68.6 \%)$ & $183(66.8 \%)$ & -0.039 \\
\hline Oxygen use & 1249 (61.3\%) & 228 (83.2\%) & 0.430 & 219 (79.9\%) & $228(83.2 \%)$ & -0.027 \\
\hline
\end{tabular}


Table 3 Subgroup analysis: baseline demographics of patients with idiopathic pulmonary fibrosis before and after propensity score matching - intensive care unit respiratory hospitalizations requiring mechanical ventilation

\begin{tabular}{|c|c|c|c|c|c|c|}
\hline & \multicolumn{3}{|c|}{ Before propensity score matching } & \multicolumn{3}{|c|}{ After propensity score matching } \\
\hline & No $\operatorname{Rx}(N=751)$ & $\begin{array}{l}\text { Pirfenidone/ } \\
\text { Nintedanib }(\mathrm{N}=94)\end{array}$ & Std. Diff & No $\operatorname{Rx}(\mathrm{N}=94)$ & $\begin{array}{l}\text { Pirfenidone/ } \\
\text { Nintedanib }(\mathrm{N}=94)\end{array}$ & Std. Diff \\
\hline \multicolumn{7}{|l|}{ Age group } \\
\hline $45-64$ & $119(15.8 \%)$ & $15(16.0 \%)$ & 0.003 & $19(20.2 \%)$ & $15(16.0 \%)$ & -0.111 \\
\hline $65-74$ & $248(33.0 \%)$ & $46(48.9 \%)$ & 0.328 & $5(47.9 \%)$ & $46(48.9 \%)$ & 0.021 \\
\hline $75+$ & $384(51.1 \%)$ & $33(35.1 \%)$ & -0.328 & $30(31.9 \%)$ & $33(35.1 \%)$ & -0.068 \\
\hline \multicolumn{7}{|l|}{ Gender } \\
\hline Female & $318(42.3 \%)$ & $26(27.7 \%)$ & -0.312 & $27(28.7 \%)$ & $26(27.7 \%)$ & -0.024 \\
\hline Male & $433(57.7 \%)$ & $68(72.3 \%)$ & 0.312 & $67(71.3 \%)$ & $68(72.3 \%)$ & 0.024 \\
\hline \multicolumn{7}{|l|}{ Race } \\
\hline White & $470(62.6 \%)$ & $58(61.7 \%)$ & -0.018 & $61(64.9 \%)$ & $58(61.7 \%)$ & -0.066 \\
\hline Black & $75(10.0 \%)$ & $6(6.4 \%)$ & -0.132 & $3(3.2 \%)$ & $6(6.4 \%)$ & 0.150 \\
\hline Hispanic & $87(11.6 \%)$ & $15(16.0 \%)$ & 0.127 & $13(13.8 \%)$ & $15(16.0 \%)$ & 0.060 \\
\hline Other & $119(15.8 \%)$ & $15(16.0 \%)$ & 0.003 & $17(18.1 \%)$ & $15(16.0 \%)$ & -0.057 \\
\hline \multicolumn{7}{|l|}{ Census region } \\
\hline Midwest & $178(23.7 \%)$ & $27(28.7 \%)$ & 0.114 & $30(31.9 \%)$ & $27(28.7 \%)$ & -0.069 \\
\hline Northeast & $113(15.0 \%)$ & $9(9.6 \%)$ & -0.167 & $12(12.8 \%)$ & $9(9.6 \%)$ & -0.101 \\
\hline South & $402(53.5 \%)$ & $50(53.2 \%)$ & -0.007 & $43(45.7 \%)$ & $50(53.2 \%)$ & 0.149 \\
\hline West & $58(7.7 \%)$ & $8(8.5 \%)$ & 0.029 & $9(9.6 \%)$ & $8(8.5 \%)$ & -0.037 \\
\hline \multicolumn{7}{|l|}{ Baseline comorbidities } \\
\hline Cardiac Arrhythmia & $350(46.6 \%)$ & $30(31.9 \%)$ & -0.303 & $30(31.9 \%)$ & $30(31.9 \%)$ & 0.000 \\
\hline Congestive Heart Failure & $337(44.9 \%)$ & $28(29.8 \%)$ & -0.315 & $25(26.6 \%)$ & $28(29.8 \%)$ & 0.071 \\
\hline Other Chronic Pulmonary Conditions & $604(80.4 \%)$ & $65(69.1 \%)$ & -0.261 & $70(74.5 \%)$ & $65(69.1 \%)$ & -0.118 \\
\hline Depression & $165(22.0 \%)$ & $22(23.4 \%)$ & 0.034 & $23(24.5 \%)$ & $22(23.4 \%)$ & -0.025 \\
\hline Diabetes & $310(41.3 \%)$ & $36(38.3 \%)$ & -0.061 & $38(40.4 \%)$ & $36(38.3 \%)$ & -0.043 \\
\hline Hypertension & $604(80.4 \%)$ & $65(69.1 \%)$ & -0.261 & $64(68.1 \%)$ & 65 (69.1\%) & 0.023 \\
\hline Pulmonary Circulation Disorder & $216(28.8 \%)$ & $27(28.7 \%)$ & -0.001 & $25(26.6 \%)$ & $27(28.7 \%)$ & 0.047 \\
\hline Renal Failure & $184(24.5 \%)$ & $13(13.8 \%)$ & -0.273 & $14(14.9 \%)$ & $13(13.8 \%)$ & -0.030 \\
\hline Solid Tumor without Metastasis & $119(15.8 \%)$ & $10(10.6 \%)$ & -0.154 & $9(9.6 \%)$ & $10(10.6 \%)$ & 0.035 \\
\hline Valvular Disease & $207(27.6 \%)$ & $19(20.2 \%)$ & -0.173 & $20(21.3 \%)$ & $19(20.2 \%)$ & -0.026 \\
\hline \multicolumn{7}{|l|}{ Elixhauser comorbidity index } \\
\hline Mean (SD) & $6.3(3.3)$ & $4.9(3.0)$ & -0.414 & $5.1(3.2)$ & $4.9(3.0)$ & -0.061 \\
\hline Median & 6.0 & 5.0 & - & 4.5 & 5.0 & - \\
\hline Q1, Q3 & $4.0,9.0$ & $3.0,6.0$ & - & $3.0,8.0$ & $3.0,6.0$ & - \\
\hline \multicolumn{7}{|l|}{ Nhospitalizations in baseline } \\
\hline 0 & $383(51.0 \%)$ & $61(64.9 \%)$ & 0.284 & $54(57.4 \%)$ & $61(64.9 \%)$ & 0.153 \\
\hline 1 & $211(28.1 \%)$ & $24(25.5 \%)$ & -0.058 & $25(26.6 \%)$ & $24(25.5 \%)$ & -0.024 \\
\hline $2+$ & $157(20.9 \%)$ & $9(9.6 \%)$ & -0.319 & $15(16.0 \%)$ & $9(9.6 \%)$ & -0.192 \\
\hline \multicolumn{7}{|l|}{ Year of hospitalization } \\
\hline 2015 & $176(23.4 \%)$ & $12(12.8 \%)$ & -0.280 & $10(10.6 \%)$ & $12(12.8 \%)$ & 0.066 \\
\hline 2016 & $204(27.2 \%)$ & $30(31.9 \%)$ & 0.104 & $29(30.9 \%)$ & $30(31.9 \%)$ & 0.023 \\
\hline 2017 & $200(26.6 \%)$ & $27(28.7 \%)$ & 0.047 & $31(33.0 \%)$ & $27(28.7 \%)$ & -0.092 \\
\hline 2018 & $171(22.8 \%)$ & $25(26.6 \%)$ & 0.089 & $24(25.5 \%)$ & $25(26.6 \%)$ & 0.024 \\
\hline \multicolumn{7}{|l|}{ Reason for admission } \\
\hline Diseases of respiratory system & $562(74.8 \%)$ & $47(50.0 \%)$ & -0.530 & $40(42.6 \%)$ & $47(50.0 \%)$ & 0.150 \\
\hline Diseases affecting the interstitium & $144(19.2 \%)$ & $44(46.8 \%)$ & 0.615 & $49(52.1 \%)$ & $44(46.8 \%)$ & -0.107 \\
\hline All other reasons & $45(6.0 \%)$ & $3(3.2 \%)$ & -0.134 & $5(5.3 \%)$ & $3(3.2 \%)$ & -0.106 \\
\hline Pulmonologist visit & $408(54.3 \%)$ & $80(85.1 \%)$ & 0.709 & $85(90.4 \%)$ & $80(85.1 \%)$ & -0.162 \\
\hline Smoker & $414(55.1 \%)$ & $46(48.9 \%)$ & -0.124 & $52(55.3 \%)$ & $46(48.9 \%)$ & -0.127 \\
\hline Steroid use & $420(55.9 \%)$ & $69(73.4 \%)$ & 0.371 & $69(73.4 \%)$ & $69(73.4 \%)$ & 0.000 \\
\hline Oxygen use & $484(64.4 \%)$ & $83(88.3 \%)$ & 0.433 & 74 (78.7\%) & 83 (88.3\%) & 0.053 \\
\hline
\end{tabular}


Table 4 Subgroup analysis: baseline demographics of patients with idiopathic pulmonary fibrosis before and after propensity score matching - intensive care unit respiratory hospitalizations without mechanical ventilation

\begin{tabular}{|c|c|c|c|c|c|c|}
\hline & \multicolumn{3}{|c|}{ Before propensity score matching } & \multicolumn{3}{|c|}{ After propensity score matching } \\
\hline & No $\operatorname{Rx}(\mathrm{N}=1288)$ & $\begin{array}{l}\text { Pirfenidone/ } \\
\text { Nintedanib } \\
(\mathrm{N}=180)\end{array}$ & Std. Diff & No $R x(N=180)$ & $\begin{array}{l}\text { Pirfenidone/ } \\
\text { Nintedanib } \\
(\mathrm{N}=180)\end{array}$ & Std. Diff \\
\hline \multicolumn{7}{|l|}{ Age group } \\
\hline $45-64$ & $168(13.0 \%)$ & $24(13.3 \%)$ & 0.010 & $30(16.7 \%)$ & $24(13.3 \%)$ & -0.093 \\
\hline $65-74$ & $341(26.5 \%)$ & $65(36.1 \%)$ & 0.208 & $65(36.1 \%)$ & $65(36.1 \%)$ & 0.000 \\
\hline $75+$ & $779(60.5 \%)$ & $91(50.6 \%)$ & -0.201 & $85(47.2 \%)$ & $91(50.6 \%)$ & 0.067 \\
\hline \multicolumn{7}{|l|}{ Gender } \\
\hline Female & $587(45.6 \%)$ & $52(28.9 \%)$ & -0.352 & $55(30.6 \%)$ & $52(28.9 \%)$ & -0.036 \\
\hline Male & $699(54.4 \%)$ & $128(71.1 \%)$ & 0.352 & $125(69.4 \%)$ & $128(71.1 \%)$ & 0.036 \\
\hline \multicolumn{7}{|l|}{ Race } \\
\hline White & $834(64.8 \%)$ & $121(67.2 \%)$ & 0.050 & $118(65.6 \%)$ & $121(67.2 \%)$ & 0.035 \\
\hline Black & $93(7.2 \%)$ & $5(2.8 \%)$ & -0.205 & $7(3.9 \%)$ & $5(2.8 \%)$ & -0.062 \\
\hline Hispanic & $131(10.2 \%)$ & $16(8.9 \%)$ & -0.044 & $17(9.4 \%)$ & $16(8.9 \%)$ & -0.019 \\
\hline Other & $230(17.9 \%)$ & $38(21.1 \%)$ & 0.086 & $38(21.1 \%)$ & $38(21.1 \%)$ & 0.000 \\
\hline \multicolumn{7}{|l|}{ Census region } \\
\hline Midwest & $386(30.0 \%)$ & $44(24.4 \%)$ & -0.125 & $40(22.2 \%)$ & $44(24.4 \%)$ & 0.053 \\
\hline Northeast & $173(13.4 \%)$ & $22(12.2 \%)$ & -0.037 & $27(15.0 \%)$ & $22(12.2 \%)$ & -0.081 \\
\hline South & $606(47.0 \%)$ & $93(51.7 \%)$ & 0.094 & $90(50.0 \%)$ & $93(51.7 \%)$ & 0.033 \\
\hline West & $123(9.5 \%)$ & $21(11.7 \%)$ & 0.068 & $23(12.8 \%)$ & $21(11.7 \%)$ & -0.034 \\
\hline \multicolumn{7}{|l|}{ Baseline comorbidities } \\
\hline Cardiac Arrhythmia & $542(42.1 \%)$ & $78(43.3 \%)$ & 0.027 & $80(44.4 \%)$ & $78(43.3 \%)$ & -0.022 \\
\hline Congestive Heart Failure & $508(39.4 \%)$ & $59(32.8 \%)$ & -0.137 & $63(35.0 \%)$ & $59(32.8 \%)$ & -0.047 \\
\hline Other Chronic Pulmonary Conditions & $964(74.8 \%)$ & $115(63.9 \%)$ & -0.240 & $118(65.6 \%)$ & $115(63.9 \%)$ & -0.035 \\
\hline Depression & $242(18.8 \%)$ & $29(16.1 \%)$ & -0.071 & $27(15.0 \%)$ & $29(16.1 \%)$ & 0.031 \\
\hline Diabetes & $509(39.5 \%)$ & $68(37.8 \%)$ & -0.034 & $69(38.3 \%)$ & $68(37.8 \%)$ & -0.011 \\
\hline Hypertension & $996(77.3 \%)$ & $129(71.7 \%)$ & -0.129 & $132(73.3 \%)$ & $129(71.7 \%)$ & -0.037 \\
\hline Pulmonary Circulation Disorder & $337(26.2 \%)$ & $59(32.8 \%)$ & 0.144 & $62(34.4 \%)$ & $59(32.8 \%)$ & -0.035 \\
\hline Renal Failure & $302(23.4 \%)$ & 39 (21.7\%) & -0.042 & $37(20.6 \%)$ & $39(21.7 \%)$ & 0.027 \\
\hline Solid Tumor without Metastasis & $183(14.2 \%)$ & $30(16.7 \%)$ & 0.067 & $27(15.0 \%)$ & $30(16.7 \%)$ & 0.046 \\
\hline Valvular Disease & $344(26.7 \%)$ & $39(21.7 \%)$ & -0.115 & $40(22.2 \%)$ & $39(21.7 \%)$ & -0.013 \\
\hline \multicolumn{7}{|l|}{ Elixhauser comorbidity index } \\
\hline Mean (SD) & $5.8(3.2)$ & $5.3(2.8)$ & -0.166 & $5.4(3.0)$ & $5.3(2.8)$ & -0.050 \\
\hline Median & 5.0 & 5.0 & - & 5.0 & 5.0 & - \\
\hline Q1, Q3 & $3.0,8.0$ & $3.0,7.0$ & - & $3.0,7.0$ & $3.0,7.0$ & - \\
\hline \multicolumn{7}{|l|}{ Nhospitalizations in baseline } \\
\hline 0 & 749 (58.2\%) & $120(66.7 \%)$ & 0.176 & 115 (63.9\%) & $120(66.7 \%)$ & 0.058 \\
\hline 1 & $324(25.2 \%)$ & $43(23.9 \%)$ & -0.030 & $43(23.9 \%)$ & $43(23.9 \%)$ & 0.000 \\
\hline $2+$ & $215(16.7 \%)$ & $17(9.4 \%)$ & -0.215 & $22(12.2 \%)$ & $17(9.4 \%)$ & -0.089 \\
\hline \multicolumn{7}{|l|}{ Year of hospitalization } \\
\hline 2015 & $272(21.1 \%)$ & $18(10.0 \%)$ & -0.311 & $17(9.4 \%)$ & $18(10.0 \%)$ & 0.019 \\
\hline 2016 & $294(22.8 \%)$ & $39(21.7 \%)$ & -0.025 & $36(20.0 \%)$ & $39(21.7 \%)$ & 0.041 \\
\hline 2017 & $332(25.8 \%)$ & $57(31.7 \%)$ & 0.130 & $61(33.9 \%)$ & $57(31.7 \%)$ & -0.047 \\
\hline 2018 & $390(30.3 \%)$ & $66(36.7 \%)$ & 0.135 & $66(36.7 \%)$ & $66(36.7 \%)$ & 0.000 \\
\hline \multicolumn{7}{|l|}{ Reason for admission } \\
\hline Diseases of respiratory system & $790(61.3 \%)$ & $89(49.4 \%)$ & -0.240 & $85(47.2 \%)$ & $89(49.4 \%)$ & 0.044 \\
\hline Diseases affecting the interstitium & $379(29.4 \%)$ & 88 (48.9\%) & 0.406 & $91(50.6 \%)$ & $88(48.9 \%)$ & -0.033 \\
\hline All other reasons & $119(9.2 \%)$ & $3(1.7 \%)$ & -0.399 & $4(2.2 \%)$ & $3(1.7 \%)$ & -0.040 \\
\hline
\end{tabular}


Table 4 (continued)

\begin{tabular}{|c|c|c|c|c|c|c|}
\hline & \multicolumn{3}{|c|}{ Before propensity score matching } & \multicolumn{3}{|c|}{ After propensity score matching } \\
\hline & No $R x(N=1288)$ & $\begin{array}{l}\text { Pirfenidone/ } \\
\text { Nintedanib } \\
(\mathrm{N}=180)\end{array}$ & Std. Diff & No $R x(N=180)$ & $\begin{array}{l}\text { Pirfenidone/ } \\
\text { Nintedanib } \\
(\mathrm{N}=180)\end{array}$ & Std. Diff \\
\hline Pulmonologist visit & $728(56.5 \%)$ & $145(80.6 \%)$ & 0.535 & $150(83.3 \%)$ & $145(80.6 \%)$ & -0.072 \\
\hline Smoker & $652(50.6 \%)$ & 89 (49.4\%) & -0.024 & $92(51.1 \%)$ & $89(49.4 \%)$ & -0.033 \\
\hline Steroid use & $725(56.3 \%)$ & $114(63.3 \%)$ & 0.144 & $114(63.3 \%)$ & $114(63.3 \%)$ & -0.000 \\
\hline Oxygen use & 765 (59.4\%) & $145(80.6 \%)$ & 0.431 & $143(79.4 \%)$ & $145(80.6 \%)$ & -0.041 \\
\hline
\end{tabular}

Table 5 Mortality at 30 days and end of follow-up following respiratory hospitalizations, ICU hospitalizations, and ICU hospializations with and without mechanical ventilation

\begin{tabular}{|c|c|c|c|}
\hline & Untreated & Treated & Statistical Analysis \\
\hline All respiratory hospitalizations & $N=402$ & $N=402$ & \\
\hline 30 day mortality, total (\%) & $40(10.0)$ & $41(10.2)$ & HR 0.96 (Cl 0.70-1.33), $p=0.812$ \\
\hline End of follow-up mortality, total (\%) & $192(47.8)$ & $81(20.1)$ & HR 0.59 (Cl 0.45-0.77), $p<0.001$ \\
\hline Months of follow-up mean(SD) & 10.6 (12.1) Median: 5.3 & 6.3 (8.9) Median: 2.2 & \\
\hline ICU hospitalizations & $N=274$ & $N=274$ & \\
\hline 30 day mortality, total (\%) & $45(16.4)$ & $49(17.9)$ & HR $1.05(\mathrm{Cl} 0.71-1.58) p=0.782$ \\
\hline End of follow-up mortality, total (\%) & $140(51.1)$ & $102(37.2)$ & HR $0.79(\mathrm{Cl} 0.61-1.02) p=0.075$ \\
\hline Months of follow-up mean (SD) & 8.7 (11.5) Median: 3.1 & 7.2 (10.4) Median: 2.4 & \\
\hline ICU Hospitalizations w/all mechanical ventilation & $N=94$ & $N=94$ & \\
\hline 30 day mortality, total (\%) & $29(30.9)$ & $27(28.7)$ & HR $0.91(\mathrm{Cl} 0.52-1.59) p=0.734$ \\
\hline End of follow-up mortality, total (\%) & $66(70.2)$ & $50(53.2)$ & HR $0.64(\mathrm{Cl} 0.43-0.94) p=0.021$ \\
\hline Months of Follow-up mean (SD) & 6.3 (10.7) Median: 1.18 & 4.1 (6.2) Median: 1.55 & \\
\hline ICU hospitalizations w/non-invasive mechanical ventilation & $N=60$ & $N=60$ & \\
\hline 30 day mortality, total (\%) & $13(21.7)$ & $13(21.7)$ & HR $0.94(\mathrm{Cl} 0.34-2.57) p=0.907$ \\
\hline End of follow-up mortality, tota I(\%) & $39(65.0)$ & $26(43.3)$ & HR $0.80(\mathrm{Cl} 0.43-1.47) p=0.472$ \\
\hline Months of Follow-up mean (SD) & 7.13 (11.02) Median: 2.37 & 4.73 (6.71) Median: 1.87 & \\
\hline ICU hospitalizations w/invasive mechanical ventilation & $N=34$ & $N=34$ & \\
\hline 30 day mortality, total (\%) & $10(29.4)$ & $14(41.2)$ & HR $1.78(\mathrm{Cl} 0.55-5.79) p=0.339$ \\
\hline End of follow-up mortality, total (\%) & $26(76.5)$ & $24(70.6)$ & HR $1.47(\mathrm{Cl} 0.66-3.27) p=0.345$ \\
\hline Months of Follow-up mean (SD) & 8.25 (13.95) Median: 1.18 & 3.03 (5.11) Median: 1.05 & \\
\hline ICU hospitalizations w/out mechanical ventilation & $N=180$ & $N=180$ & \\
\hline 30 day mortality, total (\%) & $21(11.7)$ & $22(12.2)$ & HR $1.00(\mathrm{Cl} 0.56-1.83) p=0.980$ \\
\hline End of follow-up mortality, total (\%) & 79 (43.9) & $52(28.9)$ & HR $0.71(C l 0.50-1.00) p=0.055$ \\
\hline Months of Follow-up mean (SD) & 10.0 (11.5) Median: 5.9 & 8.8 (11.7) Median: 3.0 & \\
\hline
\end{tabular}

centers, demonstrating very high in-hospital and short term mortality, as high as $100 \%$ when MV was utilized [14-18]. Over time, while remaining high, mortality has decreased as reported by recent multiple large cohort database studies demonstrating mortality or surrogate markers such as lung transplant falling closer to $50 \%$ [20-22]. The lower mortality rates observed in our study may possibly reflect improvements in the care of ICU patients, including advances in MV such as focused prevention of ventilator induced lung injury via use of "lungprotective ventilation" which include such factors as low tidal volume strategies, mechanical power, driving pressure, and stress index, as examples of the more nuanced approaches that may influence the lower mortality seen in our study [35-37]. Another factor that may contribute to lower ICU mortality may be lower thresholds for ICU admission among individual institutions. Increased recognition of AExIPF and advances in the delivery of respiratory support with non-invasive mechanical ventilation, including high flow oxygen, may allow avoidance of invasive $\mathrm{MV}$, but still necessitate ICU admission. It might be expected that this population of patients would 


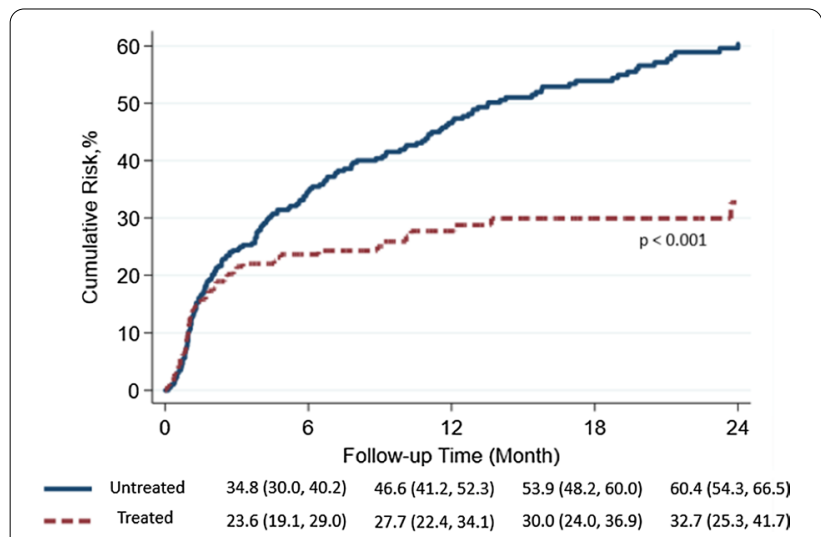

Fig. 3 Mortality cumulative risk following initial respiratory hospitalization in patients on treatment for idiopathic pulmonary fibrosis compared with untreated matched cohort

have a lower mortality than those treated with mechanical ventilation, however our findings did not suggest this, though factors that are difficult or impossible to account for such as patient preference and advanced care planning leave this an open area of interest.

In the context of our previous work on antifibrotics and treatment-related mortality, antifibrotics appear to have a role in reducing overall mortality in the first two years of therapy as well as reduce the number of hospitalizations, but do not appear to reduce in-hospital or 30-day mortality. Based on the current study findings, it would be reasonable to extrapolate that the introduction of antifibrotics during hospitalization would not affect inhospital or 30 day outcomes, though further prospective studies are needed to clarify this.

While our earlier work showed no difference in overall mortality outcomes between the two treatment options, our current work could not sufficiently evaluate this [13]. A recent analysis of Medicare beneficiaries with IPF treated with antifibrotics shortly after FDA approval found a protective effect of pirfenidone on hospitalization rates [38]. Our initial study showed lower rates of hospitalization when antifibrotic medications were used but did not directly compare this outcome between the two therapies [13]. We feel that analyzing differences between these medications to help guide clinicians in management and advances in therapy is of great interest and should be continued.

There are several limitations to our study. First, while the use of administrative billing codes has been previously used to evaluate epidemiologic outcomes of IPF, it has been noted that such methodology risks misidentification of IPF patients [39-42]. To address this limitation, we identified IPF patients using the most specific available billing codes (ICD-9 516.31 and ICD-10 J84.112) as previously described [13]. Such method importantly does not include the codes for postinflammatory fibrosis (ICD-9 515 and ICD-10 J84.10) which have previously been utilized other in studies, but not found to be specific for IPF [43]. Additionally, we attempted to remove potentially confounding or inconsistent diagnoses by removing patients with diagnostic codes for rheumatoid arthritis, hypersensitivity pneumonitis, and sarcoidosis. Another billing code limitation is that of respiratory failure which may occur in acute, chronic, or acute on chronic presentation, with coding likely to be variable across institutions and providers. An additional impact of using administrative billing codes for patient identification is that only prevalence diagnoses can be identified with accuracy. While there are likely many patients with initial, or incident, diagnoses included in our study, the potential of changing insurance coverage leaves us only able to identify the time they meet IPF diagnosis criteria based upon billing codes under their current coverage.

Another limitation is our patient population was derived from a cohort of Medicare Advantage or private insurances with pharmaceutical benefits. This could limit generalizability to patients with different or complete lack of healthcare coverage, also perhaps associated with socioeconomic risk factors that may contribute to different outcomes. Furthermore, the impact of health insurance coverage on outcomes such as mortality is difficult to evaluate, with some evidence that those on Medicare coverage may have reduced in-hospital mortality [44, 45].

A third potential limitation is the use of prescription fills as a surrogate for adherence to antifibrotic

Table 6 Utilization of intensive care unit and mechanical ventilation during initial hospitalization

\begin{tabular}{|c|c|c|c|c|c|c|}
\hline & \multicolumn{2}{|l|}{ Unmatched } & \multicolumn{2}{|l|}{ Matched } & \multirow{2}{*}{$\begin{array}{l}\text { Treated adjusted odds ratio } \\
{[95 \% \text { Confidence Interval] }}\end{array}$} & \multirow[t]{2}{*}{$p$ value } \\
\hline & Untreated $\mathrm{N}=2,511$ & Treated $\mathrm{N}=402$ & $\begin{array}{l}\text { Untreated } \\
\mathrm{N}=402\end{array}$ & Treated $\mathrm{N}=402$ & & \\
\hline $\mathrm{ICU}$ & 1385 (55.2\%) & $218(54.2 \%)$ & 224 (55.7\%) & $218(54.2 \%)$ & $0.94[0.71-1.24]$ & 0.67 \\
\hline All mechanical ventilation & $407(16.2 \%)$ & $66(16.4 \%)$ & $58(14.4 \%)$ & $66(16.4 \%)$ & $1.17[0.79-1.70]$ & 0.44 \\
\hline Non-invasive MV & $224(8.9 \%)$ & $37(9.2 \%)$ & $34(8.5 \%)$ & $37(9.2 \%)$ & $1.10[0.67-1.79]$ & 0.71 \\
\hline Invasive MV & $183(7.3 \%)$ & $29(7.2 \%)$ & $24(6.0 \%)$ & $29(7.2 \%)$ & $1.22[0.70-2.14]$ & 0.48 \\
\hline
\end{tabular}


medications. To ameliorate this, we only selected patients for the treatment arm that had continuous refilling of their prescriptions pre and post hospitalization as a surrogate of medication adherence. An additional limitation in our study is the difficulty in accurately identifying inhospital mortality as this is protected health information. Given this challenge, we instead focused on short-term mortality at 30 days noting that deaths at this point in time would have either died while hospitalized or shortly after discharge.

An interesting question yet to be answered regarding hospitalizations that our data was not able to address is the mortality of AExIPF as there is no specific billing code for this condition. It is possible that many of the respiratory events that led to hospitalization could be classified as an AExIPF, but given the lack of a diagnostic code, this was unable to be evaluated. This leaves unanswered questions about whether the antifibrotics have any impact on AExIPF mortality as well as mortality of non-AExIPF respiratory hospitalizations. Additionally, diagnostic criteria for AExIPF were revised in 2016 [46], a retrospective review of such events overlapping this time period would pose additional challenges.

\section{Conclusions}

In summary, our analysis of real-world patients with IPF hospitalized for respiratory-related conditions observed that pre-hospitalization treatment with antifibrotics had no impact on 30-day hospital-related mortality. If hospitalization was not fatal, however, ongoing treatment afterwards was associated with improved survival up to two years. Antifibrotics have previously been observed to reduce overall all-cause mortality and hospitalizations in IPF, and appear to sustain such effects even after hospitalization. While large retrospective observational studies such as ours provide broad and real-world observations, further research, particularly in the form of prospective and well documented observational studies, are necessary to identify predictive variables and interventions with a direct effect on mortality.

\begin{abstract}
Abbreviations
AExIPF: Acute exacerbation of IPF; ATS: American Thoracic Society; Cl: Confidence interval; CPT: Current procedural terminology; FDA: Federal drug administration; FVC: Forced vital capacity; HR: Hazard ratio; ICD-9: International classification of diseases, ninth edition; ICD-10: International classification of diseases, tenth edition; ICU: Intensive care unit; IPF: Idiopathic pulmonary fibrosis; MV: Mechanical ventilation; OLDW: OptumLabs data warehouse; UIP: Usual interstitial pneumonia; US: United States.
\end{abstract}

\section{Acknowledgements}

Not applicable.

\section{Authors' contributions}

B.T.K., V.T., T.M.D., L.R.S., S.R.P., T.T.T., T.M., and A.H.L. developed the concept and study design. V.T., L.R.S., S.R.P., and N.D.S. acquired and evaluated the claims data. B.T.K. drafted the initial manuscript. All authors reviewed and approved the final manuscript.

\section{Funding}

Supported by the Mayo Clinic Robert D. and Patricia E. Kern Center for the Science of Health Care Delivery, which receives no industry funding. This work was also supported by funds from Three Lakes Foundation. The sponsors of this study had no input or contributions to the development of the research and manuscript.

\section{Declarations}

\section{Ethics approval and consent to participate}

This research was conducted in accordance with all relevant guidelines and regulations. The Mayo Clinic Institutional Review Board has reviewed the use of data from the OptumLabs ${ }^{\circledR}$ Data Warehouse, a large US database with de-identified administrative claims data for individuals enrolled in private and Medicare Advantage health plans. In accordance with the Health Insurance Portability and Accountability Act and the U.S. National Institutes of Health, this research represents the use of completely-deidentified claims data. Therefore, this research is exempt from being considered human subjects research by both the Mayo Clinic Institutional Review Board and the $\mathrm{NIH}$. Since the subjects are completely de-identified, it is impossible to re-contact these individuals and hence, the need for additional informed consent is also waived by the Mayo Clinic Institutional Review Board.

\section{Consent for publication}

Not applicable.Availability of data and materials: The data that support the findings of this study are available from OptumLabs Data Warehouse but restrictions apply to the availability of these data, which were used under license for the current study, and so are not publicly available. Data are however available from the authors upon reasonable request and with permission of OptumLabs Data Warehouse.

\section{Competing interests}

The authors declare they have no competing interests.

\section{Author details}

${ }^{1}$ Department of Pulmonary and Critical Care Medicine, Mayo Clinic, Gonda 18-South, 200 1st St SW, Rochester, MN 55905, USA. ${ }^{2}$ Mayo Clinic Robert D. and Patricia E. Kern Center for the Science of Health Care Delivery, Rochester, MN, USA. ${ }^{3}$ OptumLabs, Cambridge, MA, USA. ${ }^{4}$ Division of Health Care Delivery Research, Mayo Clinic, Rochester, MN, USA.

Received: 20 March 2021 Accepted: 4 July 2021

Published online: 17 July 2021

\section{References}

1. Raghu G, Remy-Jardin M, Myers JL, Richeldi L, Ryerson CJ, Lederer DJ, et al. Diagnosis of idiopathic pulmonary fibrosis. An official ATS/ERS/JRS/ALAT clinical practice guideline. Am J Respir Crit Care Med. 2018;198(5):e44-68.

2. Nathan SD, Shlobin OA, Weir N, Ahmad S, Kaldjob JM, Battle E, et al. Longterm course and prognosis of idiopathic pulmonary fibrosis in the new millennium. Chest. 2011;140(1):221-9.

3. Ley B, Ryerson CJ, Vittinghoff E, Ryu JH, Tomassetti S, Lee JS, et al. A multidimensional index and staging system for idiopathic pulmonary fibrosis. Ann Intern Med. 2012;156(10):684-91.

4. Raghu G, Collard HR, Egan JJ, Martinez FJ, Behr J, Brown KK, et al. An official ATS/ERS/JRS/ALAT statement: idiopathic pulmonary fibrosis: evidence-based guidelines for diagnosis and management. Am J Respir Crit Care Med. 2011;183(6):788-824.

5. King TE Jr, Bradford WZ, Castro-Bernardini S, Fagan EA, Glaspole I, Glassberg MK, et al. A phase 3 trial of pirfenidone in patients with idiopathic pulmonary fibrosis. N Engl J Med. 2014;370(22):2083-92.

6. Richeldi L, du Bois RM, Raghu G, Azuma A, Brown KK, Costabel U, et al. Efficacy and safety of nintedanib in idiopathic pulmonary fibrosis. N Engl J Med. 2014;370(22):2071-82. 
7. Raghu G, Rochwerg B, Zhang Y, Garcia CA, Azuma A, Behr J, et al. An official ATS/ERS/JRS/ALAT clinical practice guideline: treatment of idiopathic pulmonary fibrosis. An update of the 2011 clinical practice guideline. Am J Respir Crit Care Med. 2015;192(2):e3-19.

8. Richeldi L, Cottin V, du Bois RM, Selman M, Kimura T, Bailes Z, et al. Nintedanib in patients with idiopathic pulmonary fibrosis: combined evidence from the TOMORROW and INPULSIS((R)) trials. Respir Med. 2016;113:74-9.

9. Lancaster L, Crestani B, Hernandez P, Inoue Y, Wachtlin D, Loaiza L, et al. Safety and survival data in patients with idiopathic pulmonary fibrosis treated with nintedanib: pooled data from six clinical trials. BMJ Open Respir Res. 2019;6(1):e000397.

10. Nathan SD, Albera C, Bradford WZ, Costabel U, Glaspole I, Glassberg MK, et al. Effect of pirfenidone on mortality: pooled analyses and meta-analyses of clinical trials in idiopathic pulmonary fibrosis. Lancet Respir Med. 2017;5(1):33-41.

11. Fisher M, Nathan SD, Hill C, Marshall J, Dejonckheere F, Thuresson PO, et al. Predicting life expectancy for pirfenidone in idiopathic pulmonary fibrosis. J Manag Care Spec Pharm. 2017;23(3-b Suppl):S17-s24.

12. Jo HE, Glaspole I, Grainge C, Goh N, Hopkins PM, Moodley Y, et al. Baseline characteristics of idiopathic pulmonary fibrosis: analysis from the Australian Idiopathic Pulmonary Fibrosis Registry. Eur Respir J. 2017:49(2):1601592.

13. Dempsey TM, Sangaralingham LR, Yao X, Sanghavi D, Shah ND, Limper AH. Clinical effectiveness of antifibrotic medications for idiopathic pulmonary fibrosis. Am J Respir Crit Care Med. 2019;200(2):168-74.

14. Blivet S, Philit F, Sab JM, Langevin B, Paret M, Guerin C, et al. Outcome of patients with idiopathic pulmonary fibrosis admitted to the ICU for respiratory failure. Chest. 2001;120(1):209-12.

15. Stern JB, Mal H, Groussard O, Brugiere O, Marceau A, Jebrak G, et al. Prognosis of patients with advanced idiopathic pulmonary fibrosis requiring mechanical ventilation for acute respiratory failure. Chest. 2001;120(1):213-9.

16. Fumeaux T, Rothmeier C, Jolliet P. Outcome of mechanical ventilation for acute respiratory failure in patients with pulmonary fibrosis. Intensive Care Med. 2001;27(12):1868-74.

17. Saydain G, Islam A, Afessa B, Ryu JH, Scott JP, Peters SG. Outcome of patients with idiopathic pulmonary fibrosis admitted to the intensive care unit. Am J Respir Crit Care Med. 2002;166(6):839-42.

18. Al-Hameed FM, Sharma S. Outcome of patients admitted to the intensive care unit for acute exacerbation of idiopathic pulmonary fibrosis. Can Respir J. 2004;11(2):117-22.

19. Brown AW, Fischer CP, Shlobin OA, Buhr RG, Ahmad S, Weir NA, et al. Outcomes after hospitalization in idiopathic pulmonary fibrosis: a cohort study. Chest. 2015;147(1):173-9.

20. Mooney JJ, Raimundo K, Chang E, Broder MS. Mechanical ventilation in idiopathic pulmonary fibrosis: a nationwide analysis of ventilator use, outcomes, and resource burden. BMC Pulm Med. 2017;17(1):84.

21. Rush B, Wiskar K, Berger L, Griesdale D. The use of mechanical ventilation in patients with idiopathic pulmonary fibrosis in the United States: a nationwide retrospective cohort analysis. Respir Med. 2016;111:72-6.

22. Durheim MT, Judy J, Bender S, Baumer D, Lucas J, Robinson SB, et al. In-hospital mortality in patients with idiopathic pulmonary fibrosis: a US cohort study. Lung. 2019;197(6):699-707.

23. Alqalyoobi S, Fernández Pérez ER, Oldham JM. In-hospital mortality trends among patients with idiopathic pulmonary fibrosis in the United States between 2013-2017: a comparison of academic and non-academic programs. BMC Pulm Med. 2020;20(1):289.

24. Wallace PJ, Shah ND, Dennen T, Bleicher PA, Crown WH. Optum Labs: building a novel node in the learning health care system. Health Aff. 2014;33(7):1187-94.

25. Mooney JJ, Raimundo K, Chang E, Broder MS. Hospital cost and length of stay in idiopathic pulmonary fibrosis. J Med Econ. 2017;20(5):518-24.

26. Quan H, Sundararajan V, Halfon P, Fong A, Burnand B, Luthi J-C, et al. Coding algorithms for defining comorbidities in ICD-9-CM and ICD-10 administrative data. Med Care. 2005;43:1130-9.
27. Pedraza-Serrano F, Jimenez-Garcia R, Lopez-de-Andres A, HernandezBarrera V, Sanchez-Munoz G, Puente-Maestu L, et al. Characteristics and outcomes of patients hospitalized with interstitial lung diseases in Spain, 2014 to 2015. Medicine (Baltimore). 2019;98(21):e15779.

28. Yao X, Gersh BJ, Holmes DR, Melduni RM, Johnsrud DO, Sangaralingham $L R$, et al. Association of surgical left atrial appendage occlusion with subsequent stroke and mortality among patients undergoing cardiac surgery. JAMA. 2018;319(20):2116-26.

29. Austin PC. Optimal caliper widths for propensity-score matching when estimating differences in means and differences in proportions in observational studies. Pharm Stat. 2011;10(2):150-61.

30. Austin PC. Balance diagnostics for comparing the distribution of baseline covariates between treatment groups in propensity-score matched samples. Stat Med. 2009;28(25):3083-107.

31. Gayat E, Resche-Rigon M, Mary JY, Porcher R. Propensity score applied to survival data analysis through proportional hazards models: a Monte Carlo study. Pharm Stat. 2012;11(3):222-9.

32. Oda K, Yatera K, Fujino Y, Kido T, Hanaka T, Sennari K, et al. Respiratory comorbidities and risk of mortality in hospitalized patients with idiopathic pulmonary fibrosis. Respir Investig. 2018;56(1):64-71.

33. Saito S, Lasky JA, Hagiwara K, Kondoh Y. Ethnic differences in idiopathic pulmonary fibrosis: the Japanese perspective. Respir Investig. 2018;56(5):375-83.

34. Hutchinson J, Fogarty A, Hubbard R, McKeever T. Global incidence and mortality of idiopathic pulmonary fibrosis: a systematic review. Eur Respir J. 2015;46(3):795-806.

35. Slutsky AS, Ranieri VM. Ventilator-induced lung injury. N Engl J Med. 2013;369(22):2126-36.

36. Tonetti T, Vasques F, Rapetti F, Maiolo G, Collino F, Romitti F, et al. Driving pressure and mechanical power: new targets for VILI prevention. Ann Transl Med. 2017;5(14):286.

37. Cortes-Puentes GA, Holets SR, Oeckler RA. Integration of pulmonary mechanics in a personalized approach to mechanical ventilation. Respir Care. 2018;63(9):1194-6.

38. Corral M, Chang E, Broder MS, Gokhale S, Reddy SR. Healthcare use and costs among Medicare enrollees on pirfenidone versus nintedanib for idiopathic pulmonary fibrosis. J Comp Effect Res. 2020;9(13):933-43.

39. Collard HR, Chen SY, Yeh WS, Li Q, Lee YC, Wang A, et al. Health care utilization and costs of idiopathic pulmonary fibrosis in U.S. Medicare beneficiaries aged 65 years and older. Ann Am Thorac Soc. 2015;12(7):981-7.

40. Collard HR, Ward AJ, Lanes S, Cortney Hayflinger D, Rosenberg DM, Hunsche E. Burden of illness in idiopathic pulmonary fibrosis. J Med Econ. 2012;15(5):829-35.

41. Raghu G, Weycker D, Edelsberg J, Bradford WZ, Oster G. Incidence and prevalence of idiopathic pulmonary fibrosis. Am J Respir Crit Care Med. 2006;174(7):810-6.

42. Raghu G, Chen SY, Hou Q, Yeh WS, Collard HR. Incidence and prevalence of idiopathic pulmonary fibrosis in US adults 18-64 years old. Eur Respir J. 2016;48(1):179-86.

43. Vu A, Vasireddy A, Moua T, Baqir M, Ryu JH. Clarifying the diagnosis of post-inflammatory pulmonary fibrosis: a population-based study. Eur Respir J. 2019;54(1):1900103.

44. Sommers BD, Gawande AA, Baicker K. Health insurance coverage and health — what the recent evidence tells us. N Engl J Med. 2017;377(6):586-93.

45. Card D, Dobkin C, Maestas N. Does medicare save lives? Q J Econ. 2009;124(2):597-636.

46. Collard HR, Ryerson CJ, Corte TJ, Jenkins G, Kondoh Y, Lederer DJ, et al. Acute exacerbation of idiopathic pulmonary fibrosis. An international working group report. Am J Respir Crit Care Med. 2016;194(3):265-75.

\section{Publisher's Note}

Springer Nature remains neutral with regard to jurisdictional claims in published maps and institutional affiliations. 